\title{
VLBA ABSORPTION IMAGING OF IONIZED GAS ASSOCIATED WITH THE ACCRETION DISK IN NGC 1275
}

\author{
R. C. Walker, V. Dhawan, and J. D. Romney \\ National Radio Astronomy Observatory, Socorro, NM 87801 \\ K. I. Kellermann \\ National Radio Astronomy Observatory, Charlottesville, VA 22901 \\ R. C. Vermeulen \\ Netherlands Foundation for Radio Astronomy, Dwingeloo, The Netherlands
}

Accepted 1999 Sept. 9 for publication in the Astrophysical Journal.

\begin{abstract}
Nearly simultaneous VLBA observations of $3 \mathrm{C} 84$, the radio source associated with NGC 1275, have been made at multiple frequencies to study the free-free absorption of the northern, or "counterjet", feature found by Walker, Romney, and Benson (1994) and by Vermeulen, Readhead, and Backer (1994). Our observations confirm that the spectra are consistent with free-free absorption and eliminate the possibility that the earlier result was an effect of variability. The northern feature is well resolved spatially, so images have been made showing the distribution of the absorption over a region of about $1.5 \mathrm{pc}$ on a side, beginning about $1.5 \mathrm{pc}$ from the presumed location of the central object. That distribution is dominated by a strong decrease with radial distance. The magnitude of the absoption near $2.5 \mathrm{pc}$ projected distance from the central object is consistent with a $10^{4} \mathrm{~K}$ gas with an emission measure of about $5 \times 10^{8}$ pc $\mathrm{cm}^{-6}$. The geometry is consistent with absorption by ionized gas associated with an accretion disk. The data provide firm constraints for models of the outer regions of accretion disks and, perhaps, associated winds.
\end{abstract}

Subject headings: galaxies: individual(NGC 1275) — galaxies:jets — galaxies: active — radio continuum: galaxies 


\section{Introduction}

The dominant member of the Perseus Cluster is the galaxy NGC 1275, which has a Seyfert-like nucleus, but an unusual morphology. Minkowski (1957) found that it contains two velocity systems, one at $5300 \mathrm{~km} \mathrm{~s}^{-1}$ and the other at $8200 \mathrm{~km} \mathrm{~s}^{-1}$. The low velocity system includes the main body of NGC 1275 (Strauss et al. 1992) and filaments of ionized gas thought to be a product of the cooling flow that is centered on the galaxy. CO and X-ray observations suggest the cooling flow rate is several hundred $\mathrm{M}_{\odot} \mathrm{yr}^{-1}$ (Lazareff et al. 1989; Allen \& Fabian 1997). HST and Keck observations of globular clusters suggest that many may have been formed in a merger event several hundred million years ago (Carlson et al. 1998; Brodie et al. 1998). The high velocity system includes giant H II clouds. Hydrogen associated with the high velocity system is seen in absorption against the nuclear radio emission (DeYoung, Roberts, \& Saslaw 1973; Romney 1978). This and other evidence indicate that the high velocity system is either infalling, or is a system that is already part way through the main galaxy (e.g. Kaisler et al. 1996; Nørgaard-Nielsen et al. 1993).

The radio source $3 \mathrm{C} 84$ is associated with NGC 1275. The features coincident with the center of the galaxy constitute one of the strongest compact radio sources in the sky. This source began a major increase in activity in about 1959. It was below $10 \mathrm{Jy}$ in the earliest observations, but was already rising towards peaks in excess of 50 Jy in the 1970's and 1980's (Dent 1966; Pauliny-Toth \& Kellermann 1966; Pauliny-Toth et al. 1976; O’Dea, Dent, and Balonek 1984; Nesterov, Lyuty, \& Valtaoja 1995). It has been high ever since, although in recent years the flux density had been decaying and is currently around $20 \mathrm{Jy}$ at centimeter wavelengths.

The compact structure of 3C 84 has been the object of Very Long Baseline Interferometry (VLBI) observations since the early days of that technique (See, for example, Pauliny-Toth et al. 1976; Readhead et al. 1983; Romney et al. 1984; Marr et al. 1989; Biretta, Bartel, \& Deng 1991; Krichbaum et al. 1993; Venturi et al. 1993; Dhawan, Kellermann, and Romney 1998; Romney, Kellermann, \& Alef 1999). It has complex structure on parsec scales that frustrated imaging efforts in the early years. But early models, combined with more recent images made with the global networks and with the Very Long Baseline Array (VLBA - Napier et al. 1994) show that, south of the compact core, there is a bright region of emission that has been increasing in length

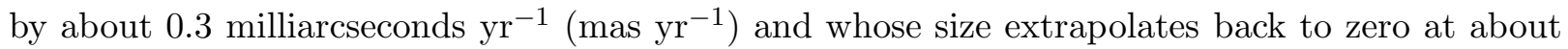
the time of the 1959 outburst (Romney et al. 1982; the rate is from Walker, Romney, and Benson 1994, hereafter WRB, and is based on their image and others from the literature). The bright region now extends about 15 mas south of the compact core. It is almost certainly related to a jet, but the morphology is somewhat like the radio lobes seen in many sources on much larger scales.

This may indicate that the jet material from the 1959 event is not simply following earlier material down the jet channel, but is interacting strongly with either that material or the surrounding medium. To avoid a physical bias, we will simply call the bright regions "features". For this study, they are only used as sources of background radiation with which to study the absorbing medium. 
Beyond the bright southern feature, the remnants of earlier activity are clearly seen. Immediately south of the 15 mas bright feature, low frequency VLBI observations show a continuation of the jet to about 100 mas (e.g., Taylor \& Vermeulen 1996; Silver, Taylor, \& Vermeulen 1998, hereafter STV; this paper). STV also see a "millihalo" approximately 250 mas in size at $330 \mathrm{MHz}$. On scales of arcseconds and larger, 3C 84 has weak, somewhat asymmetric twin jet structure surrounded by a diffuse halo (Pedlar et al. 1990; Sijbring 1993).

In 1993, a feature extending to about 8 mas north of the compact core was discovered with the VLBA at $8.4 \mathrm{GHz}$ by WRB and independently, using 1991 Global VLBI Network data at 22 GHz, by Vermeulen, Readhead, and Backer (1994, hereafter VRB). At 22 GHz, this feature was similar to, but smaller and somewhat weaker than the bright southern feature. It most likely is related to a jet on the far side of the AGN corresponding to the near side jet responsible for the southern feature. This source provides an especially favorable case for relating jet and counterjet features because of the dramatic changes in jet brightness dating to the 1959 outburst. WRB show that the ratio of distances to the ends of the bright emission in the north and south, the brightness ratio of the features at $22 \mathrm{GHz}$, and the measured speed of the southern feature, are consistent with a mildly relativistic jet $(0.3 c$ to $0.5 c)$ at a moderate angle $\left(30^{\circ}\right.$ to $\left.55^{\circ}\right)$ to the line-of-sight.

The most exciting result of WRB and VRB was the observation that the northern feature has a strongly inverted spectral index. It was very much brighter at $22 \mathrm{GHz}$ than at $8.4 \mathrm{GHz}$, and it had not been seen at all in high dynamic range images of Biretta et al. (1991) at $1.7 \mathrm{GHz}$. But the 22 and $8.4 \mathrm{GHz}$ observations were separated in time by about 2 years so there was some potential for confusion due to time dependent effects. VRB present persuasive arguments that the spectral index, if confirmed, is most likely the result of free-free absorption by ionized material along the line-of-sight to the northern feature. This absorbing material must be located in the inner few parsecs of the source - otherwise it is highly unlikely that it would absorb only the northern feature leaving the core and southern feature unaffected. An obvious geometry consistent with the data puts the ionized gas in, or associated with, a disk. Levinson, Laor, and Vermeulen (1995, hereafter LLV) discuss models involving accretion disks at some length. One problem is that, according to simple theory, the accretion disk should be too cold at parsec distances to contain ionized gas. They address a variety of mechanisms that would allow ionizing radiation from the central regions of the AGN to reach the outer disk and create an ionized atmosphere adequate to explain the observed absorption.

Apparent free-free absorption is also seen in this source at lower frequencies. O'Dea, Dent, and Balonek (1984) deduce, from flux density monitoring data, that the variable core component is absorbed below about $2.2 \mathrm{GHz}$. They suggest that the absorbing gas has a thickness of $1.5<L<5 \mathrm{pc}, T \sim 10^{4} \mathrm{~K}$, and $n \sim 2 \times 10^{3} \mathrm{~cm}^{-3}$. More recently, STV found a feature 80 mas north of the core in 330, 612, and $1414 \mathrm{MHz}$ VLBA observations that presumably corresponds to an earlier epoch of activity. The spectrum of this feature can be interpreted in terms of free-free absorption on a much larger scale. 
Evidence for free-free absorption on parsec scales has been seen in a few other sources. Jones et al. (1996) find preferential absorption of counterjet emission in Cen A, indicating a geometry similar to what is seen in 3C 84. Jones and Wehrle (1997) see a feature in NGC 4261 that is possibly due to absorption by an inner accretion disk with a width of less than $0.1 \mathrm{pc}$, but they do not have spectral information on this feature. Kellermann, Vermeulen, Cohen, and Zensus (1999) report the presence of free-free absorption of the nucleus and receding jet in NGC 1052, with the absorption moving to lower frequencies over scales from 15 light days to more than 3 light years. Taylor (1996) attributes the spectral turnover seen in the parsec scale jet components in Hydra A to free-free absorption over the entire central region, probably due to gas on a somewhat larger scale. Parsec scale ionized gas associated with a disk may have been observed directly in NGC1068 by Gallimore, Baum, \& O'Dea (1997). The inferred temperature of the observed region is around $10^{6.7} \mathrm{~K}$. But the case that this is ionized disk gas, rather than a jet, is not firmly established.

Ulvestad, Wrobel, \& Carilli (1999) report evidence for absorption in Markarian 231, a powerful Seyfert 1 starburst galaxy at $z=0.0422$ whose compact emission regions look remarkably similar to $3 \mathrm{C}$ 84. In both magnitude and physical scale, the observed absorption in Mrk 231 is more comparable to that observed in $3 \mathrm{C} 84$ by STV than that reported in this paper. Unlike 3C 84, a spectral gradient is reported for the southern feature, implying some absorption over the whole source. But this may be a result of alignment of the images on the core. At least in $3 \mathrm{C} 84$, the centroid of the core shifts with frequency. Also, the $1.4 \mathrm{GHz}$ image shows emission well north of any seen at higher frequencies, implying unphysically steep spectral indices or some imaging effect such as much greater sensitivity to large structures at $1.4 \mathrm{GHz}$ than at the other frequencies.

In this paper, we present results of the first two epochs of observations of 3C 84 designed to study the absorption and its two dimensional distribution. The observations were made with the Very Long Baseline Array of the National Radio Astronomy Observatory, supplemented in some cases by a single antenna of the Very Large Array (VLA). At each epoch, a wide range of frequencies were observed, all within a period short enough to preclude uncertainties due to variability. High dynamic range images, all convolved to the same resolution, allow us to image the absorption over the extent of the northern feature.

The first, and simplest, result is that the apparent absorption is not due to temporal changes in the source. The quasi-instantaneous radio spectra at positions on the northern feature are consistent with free-free absorption. Minor deviations from a theoretical free-free spectrum are probably due to inhomogeneities in the medium. We show that the dominant spatial structure of the absorption is a strong decrease with radial distance from the central feature. The concept that the ionized gas is associated with the accretion disk and falls off in density from the center is consistent with the observations.

At $v=5300 \mathrm{~km} \mathrm{~s}^{-1}, 1$ mas $=0.25 h^{-1} \mathrm{pc}$, where $H_{o}=100 h \mathrm{~km} \mathrm{~s}^{-1} \mathrm{Mpc}^{-1}$. In this paper, we assume that $h=0.75$, so the scale of the images is very close to $1 \mathrm{pc}=3$ mas. 


\section{The Observations}

Observations of 3C84 were made with the VLBA in 1995 January and October at 2.3, 5.0, 8.4, 15.4, 22, and $43 \mathrm{GHz}$. The specific dates are listed in Table 1. The observations were spread over several days at each epoch in order to gather sufficient data at each frequency to make high dynamic range images. However all observations for each epoch were confined to a period of two weeks in order to avoid confusion by possible time variations. A single antenna of the VLA was included in the 22 and $43 \mathrm{GHz}$ observations in October in order to enhance the coverage of short baselines. The day following the last of our October observations, 3C 84 was observed at 0.33, 0.61, and $1.4 \mathrm{GHz}$ by STV. Therefore near simultaneous observations of this source in 1995 October are available at all bands for which the VLBA has receivers.

The northern feature seen at higher frequencies about 8 mas from the core was not detectable at $2.3 \mathrm{GHz}$ to a limit of about $2 \mathrm{mJy}_{\text {beam }}{ }^{-1}$ in both January and October, presumably because it is too heavily absorbed. Also the much more distant northern feature, at about 80 mas, that was reported by STV at low frequencies, was not seen in our $2.3 \mathrm{GHz}$ images, perhaps because of inadequate short baselines. The convolved images used to look for the distant features have off source noise levels of about $1.5 \mathrm{mJy}^{\text {beam }^{-1}}$ with beams of 19 by 15 mas. At $43 \mathrm{GHz}$ the the feature 8 mas north of the core was detected, but most of the flux density appears to be missing. This feature is several mas in size, which is a size scale that falls in the range that is poorly measured at $43 \mathrm{GHz}$ because of the gap in $(\mathrm{u}, \mathrm{v})$ coverage between the VLBA and the VLA. The feature is mostly resolved out on the VLBA, but is still a point source to the VLA. Therefore the results presented here will be based almost entirely on the 5.0, 8.4, 15.4, and $22 \mathrm{GHz}$ images, although we show the $2.3 \mathrm{GHz}$ images and one $43 \mathrm{GHz}$ image.

Details of the observations are presented in Table 1. The columns are the date within 1995, the antennas included, the bit rate, the polarizations recorded, and the number of effective baseline hours of data available for the final image. The total recorded bit rate was divided among 2 , 4, or 8 baseband channels. The baseline hours listed is simply the integration time times the number of visibility records. The $2.3,5.0$, and $8.4 \mathrm{GHz}$ observations were performed by band switching during a single day in both January and October. In January, but not in October, the 2.3 and 8.4 $\mathrm{GHz}$ data were obtained simultaneously using the VLBA's dichroic system.

Apriori amplitude calibration was done in the usual way using the system temperatures measured during the observations and gains measured by the NRAO staff during pointing observations. Most of the data were processed with AIPS. The $22 \mathrm{GHz}$ image from January was made using DIFMAP (Shepherd, Pearson, \& Taylor 1994). On 1995 October 20, VLA observations were made of $3 \mathrm{C} 84$ and the compact source DA $193(0552+398)$ to obtain flux densities. These were used to check and adjust the apriori amplitude calibration of the VLBA. Adjustments in the amplitude scales by factors of 1.00, 0.91, 1.00, 0.95 for 22, 15.4, 8.4, and 5.0 GHz were made as a result. The remaining uncertainty in the flux scale is roughly estimated to be about $5 \%$ for the October data. For January, the apriori calibration had to be trusted and there may be residual 
Table 1. VLBA Observations of 3C 84

\begin{tabular}{|c|c|c|c|c|c|}
\hline $\begin{array}{l}\text { Date } \\
(1995)\end{array}$ & $\begin{array}{c}\text { Frequency } \\
\quad(\mathrm{GHz})\end{array}$ & Antennas & $\begin{array}{c}\text { Bit Rate } \\
\text { (Mbps) }\end{array}$ & Polarization & $\begin{array}{c}\text { Baseline } \\
\text { Hours }\end{array}$ \\
\hline Jan 15 & 22.2 & VLBA & 64 & dual & 384 \\
\hline \multirow[t]{3}{*}{ Jan 22} & $2.3^{\mathrm{ab}}$ & $\mathrm{VLBA}^{\mathrm{c}}$ & 32 & dual & 159 \\
\hline & 5.0 & $\mathrm{VLBA}^{\mathrm{c}}$ & 64 & dual & 88 \\
\hline & $8.4^{\mathrm{b}}$ & VLBA $^{c}$ & 32 & dual & 158 \\
\hline Jan 29 & 15.3 & VLBA & 64 & LCP & 249 \\
\hline Jan 30 & 43.2 & VLBA & 128 & $\mathrm{LCP}$ & 399 \\
\hline Oct 09 & 15.3 & VLBA & 128 & dual & 174 \\
\hline Oct $16^{\mathrm{d}}$ & 43.2 & $\mathrm{VLBA}+\mathrm{VLA}^{\mathrm{e}}$ & 128 & LCP & \\
\hline Oct 21 & 22.2 & $\mathrm{VLBA}+\mathrm{VLA}^{\mathrm{e}}$ & 128 & dual & 244 \\
\hline \multirow[t]{3}{*}{ Oct 22} & $2.3^{\mathrm{a}}$ & VLBA & 64 & dual & 44 \\
\hline & 5.0 & VLBA & 64 & dual & 116 \\
\hline & 8.4 & VLBA & 64 & dual & 135 \\
\hline Oct $23 \mathrm{df}$ & $0.3,0.6,1.4$ & VLBA & 64 & dual & \\
\hline
\end{tabular}

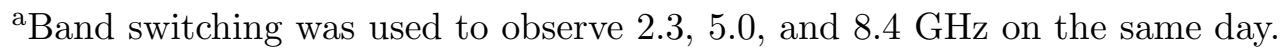

${ }^{\mathrm{b}}$ Dichroic system used to observe 2.3 and $8.4 \mathrm{GHz}$ simultaneously on Jan. 22 .

${ }^{\mathrm{c}}$ Only 9 of the 10 VLBA antennas participated.

${ }^{\mathrm{d}}$ Data not used for this paper, but listed for completeness.

e Included one antenna only of the VLA.

${ }^{\mathrm{f}}$ Independent but related project (Silver, Taylor, \& Vermeulen 1998). 


\section{Jan 95}

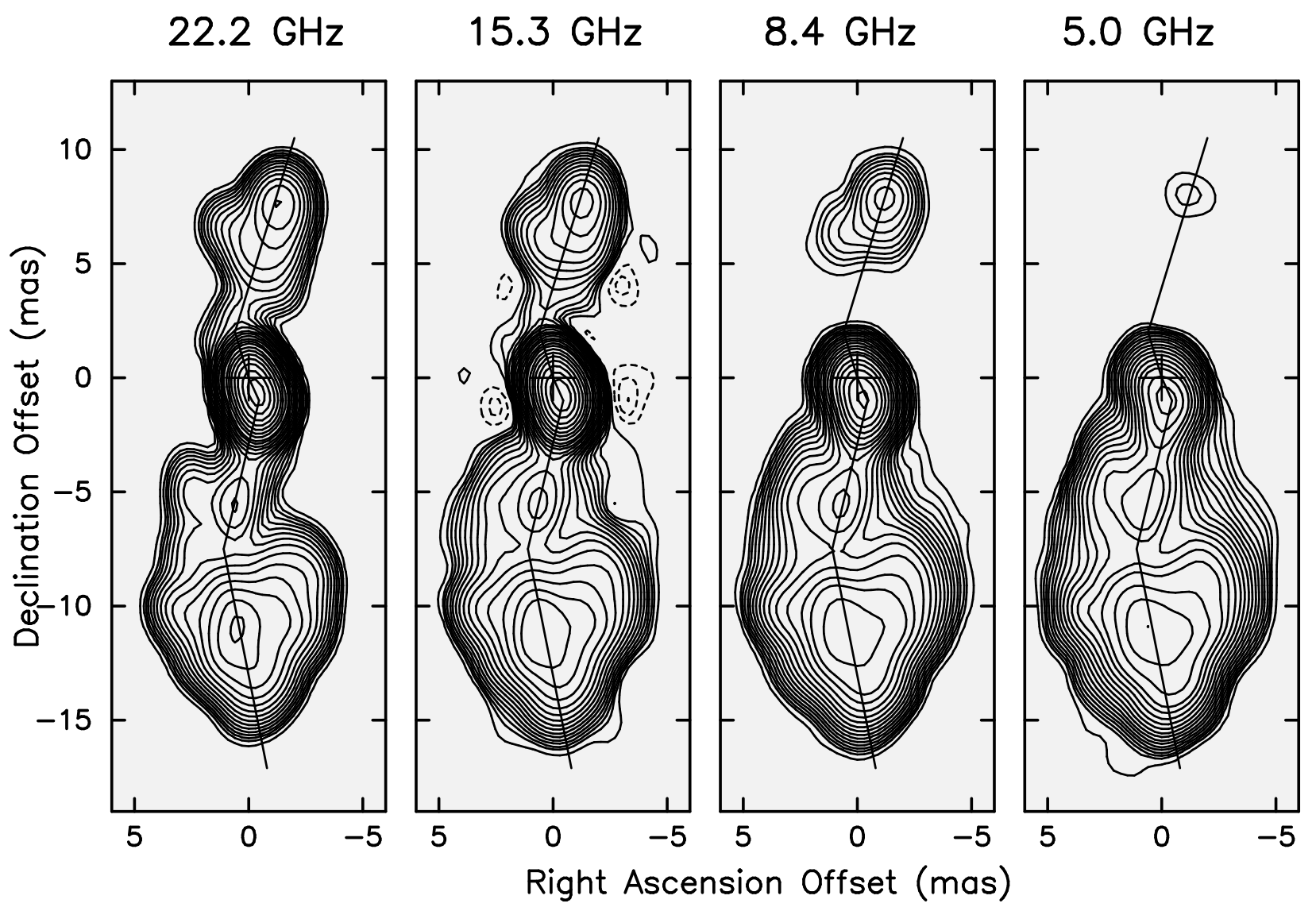

Fig. 1.- Montage of the VLBA images of 3C 84 based on data taken in 1995 January. These displays are based on the CLEAN components convolved with a common Gaussian beam of 1.6 by 1.2 mas, elongated north-south. The contour levels start with 5, 10, 14, $20 \mathrm{mJy} \mathrm{beam}^{-1}$ and increase from there by factors of $\sqrt{2}$. The residual image is not included, but the CLEANs are sufficiently deep, and the lowest contour sufficiently high, that this does not have a significant effect. The north-south segmented line shows the location of the slice along which some of the analysis was done. Note that, for $H_{o}=75 \mathrm{~km} \mathrm{~s}^{-1} \mathrm{Mpc}^{-1}$, the scale is about 3 mas per parsec.

errors at the $10 \%$ level. The $2.3 \mathrm{GHz}$ amplitudes from January were scaled by 0.87 to adjust for the effects of an inappropriate off-source position used in VLBA 2.3/8.4 GHz dual frequency gain measurements prior to 1997 November.

Montages of the contour plots of the images used in the analysis are shown in Figures 1 and 2. All images are convolved to a beam of 1.6 by 1.2 mas, elongated in position angle 0 degrees. This is about the actual resolution of the $5 \mathrm{GHz}$ images, but represents a considerable smoothing of the higher frequency images. On each contour plot, a segmented line is shown which marks the slice used for some of the analysis below. 


\section{Oct 95}

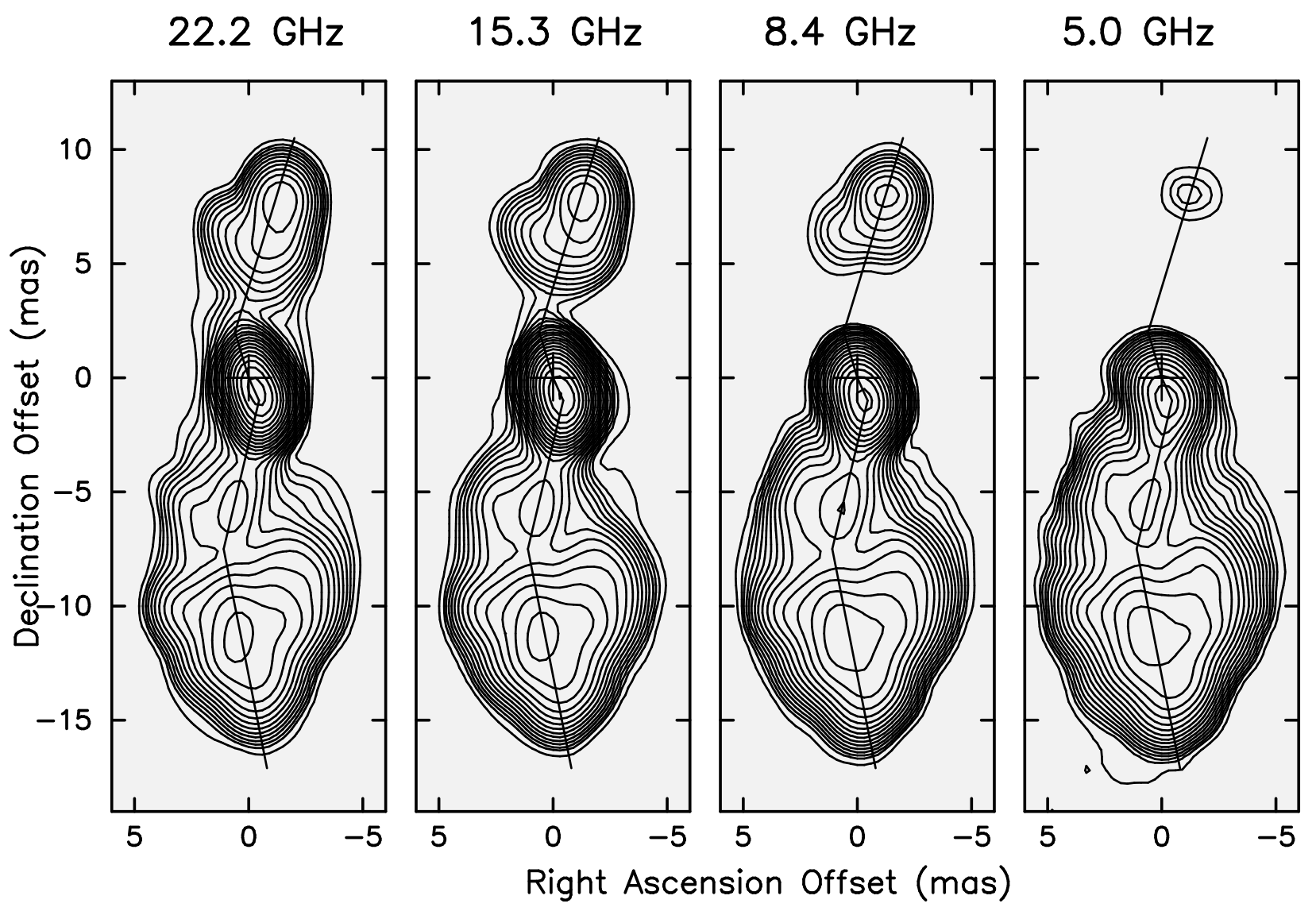

Fig. 2.- Montage of the VLBA images of 3C 84 based on data taken in 1995 October. The beam, contour levels and slice are the same as for Figure 1 .

Contour plots of the 2.3 GHz images are shown in Figure 3. The resolution of these images is lower than for the others because of the lower frequency. These images show the southern feature well and make it clear that there is a weak, underlying structure extending well beyond the region of the features that resulted from the 1959 outburst. Those underlying structures are also seen weakly in the $5 \mathrm{GHz}$ images, although only when plotted to deeper levels than were used in Figures 1 and 2. The northern feature is absent, presumably completely absorbed. In the 1995 October image at $22 \mathrm{GHz}$, the ratio of integrated flux density in the northern and southern features is about 3.7. In the $2.3 \mathrm{GHz}$ image from the same epoch, the integrated flux density of the southern feature is about $21 \mathrm{Jy}$. If the intrinsic spectral index (without absorption) of the northern and southern features is the same, the expected flux density of the northern feature at $2.3 \mathrm{GHz}$ would be $5.7 \mathrm{Jy}$. Instead it is below about $2 \mathrm{mJy}$, so there is very strong absorption corresponding to an optical depth of at least 7.9. At $5 \mathrm{GHz}$, the northern feature had an integrated flux density of about $20 \mathrm{mJy}$ in January and $34 \mathrm{mJy}$ in October in a region smaller than the 2.3 

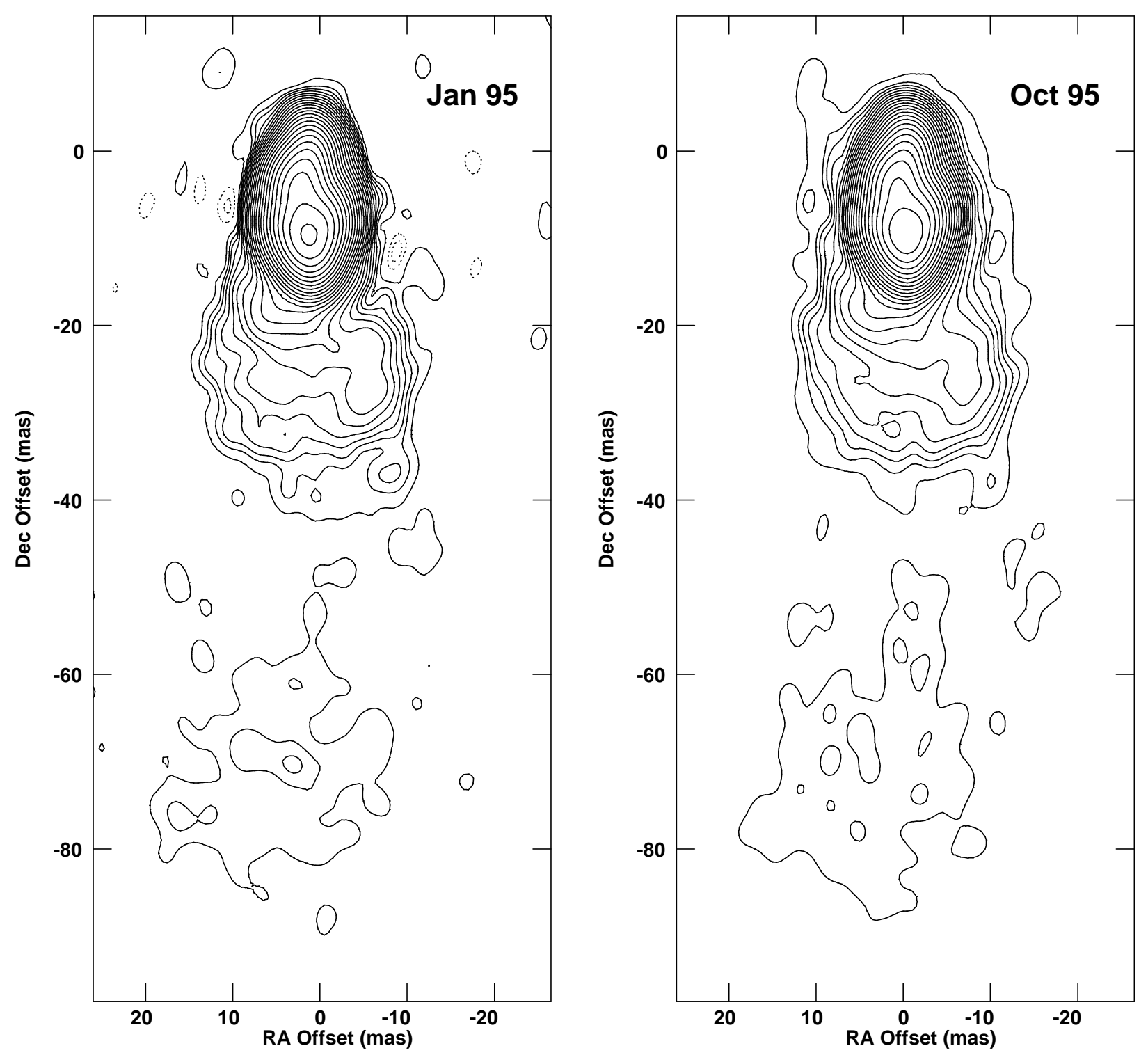

Fig. 3.- The images of 3C 84 at $2.3 \mathrm{GHz}$ from 1995 January and 1995 October. The convolving beams are $4.4 \times 3.2$ mas elongated north-south. These are fully restored CLEAN images - they include the residuals, unlike the images in Figures 1 and 2. The contours are 2, 4, 5.7, 8, mJy beam $^{-1}$ increasing from there by factors of the $\sqrt{2}$. The peaks in the images are $8.84 \mathrm{Jy} \mathrm{beam}^{-1}$ in January and $7.90 \mathrm{Jy}_{\text {beam }}^{-1}$ in October. No effort has been made to carefully align these images either with each other or with those of Figures 1 and 2 . Note the scales - these images show emission well south of what is seen at higher frequencies. There clearly have been previous outbursts of this source. 


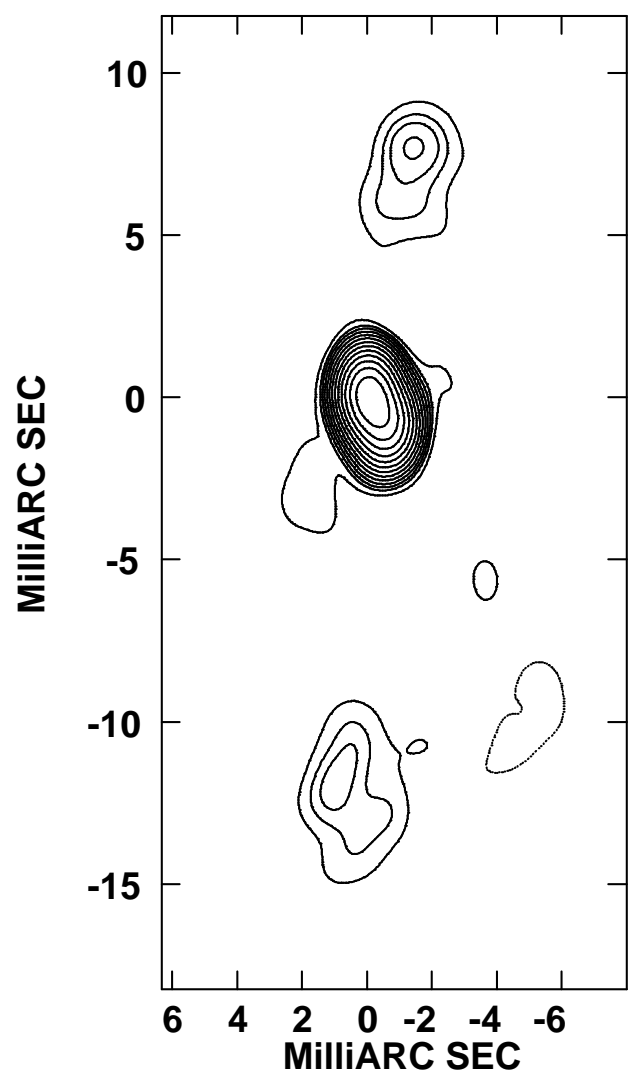

Fig. 4.- This image shows the results at $43 \mathrm{GHz}$ from 1995 January, convolved to the same resolution as Figures 1 and 2. The lowest contour levels are 20 and $40 \mathrm{mJy} \mathrm{beam}^{-1}$, increasing by factors of $\sqrt{2}$ from there. It is clear that most of the flux density from the northern and southern features is missing in these images. These structures fall in a size range that, at $43 \mathrm{GHz}$, is still unresolved by the VLA but is almost completely resolved out by the shortest VLBA baselines. Therefore, this image is only used to demonstrate that the features are present at $43 \mathrm{GHz}$, but not to place any constraints on them.

$\mathrm{GHz}$ beam. Thus the observed spectral index between 2.3 and $5.0 \mathrm{GHz}$ was at least 2.9 in January and 3.6 in October. While we do not use this information in the following analysis, it further supports the interpretation of the spectra as being due to the exponential cutoff resulting from free-free absorption toward the northern feature.

An image based on the $43 \mathrm{GHz}$ VLBA data from Jan. 1995 is shown in Figure 1 . The image has been convolved to the same beam used in the analysis of the 5 to $22 \mathrm{GHz}$ images. The northern and southern features are clearly there, but at much reduced flux densities because of the lack of baselines of a few tens to a few hundred $\mathrm{km}$. Because of this missing flux density, this image cannot be used in the analysis. For more on VLBA monitoring of the $43 \mathrm{GHz}$ structure of 


\section{Oct 95}

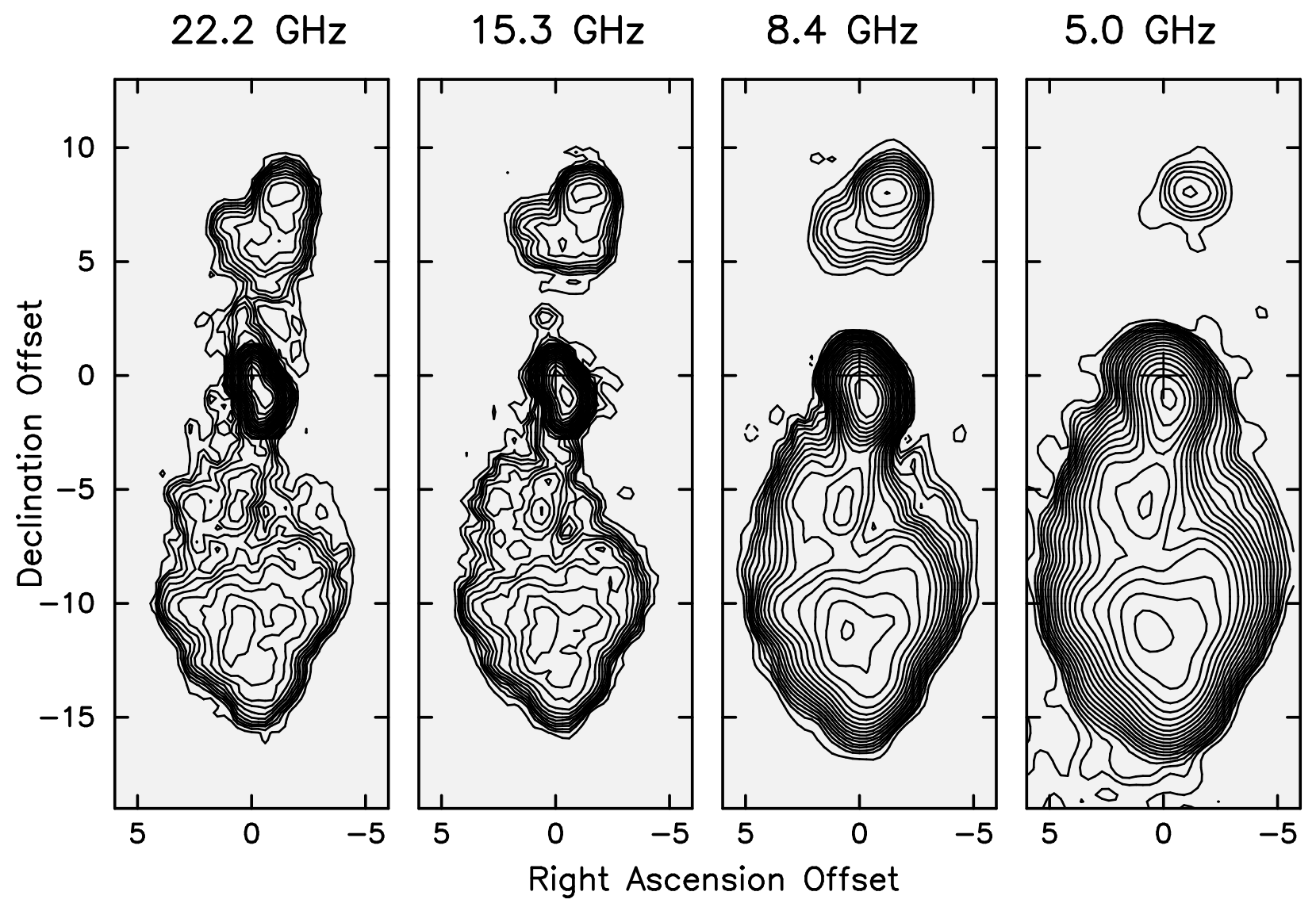

Fig. 5.- Montage of full resolution images of 3C 84 from 1995 October. The displays are based on the same CLEAN components as were used for Figure 2 2, but using the full resolution of the original images. The convolving beams are (major axis in mas, minor axis in mas, position angle in degrees) $(0.73,0.48,-2.90)$ for $22 \mathrm{GHz},(0.68,0.50,2.93)$ for $15 \mathrm{GHz},(1.21,0.88,-2.61)$ for 8.4 $\mathrm{GHz}$, and $(1.6,1.2,0.0)$ for $5.0 \mathrm{GHz}$. The lowest contour levels are 2 and $4 \mathrm{mJy}^{\mathrm{beam}}{ }^{-1}$, increasing by factors of $\sqrt{2}$ from there. The beams, especially the $22 \mathrm{GHz}$ beam, don't scale with frequency because different tapers and weighting schemes were used.

this source, see Dhawan, Kellermann, and Romney (1998).

For background information, full resolution images of the source in 1995 October are shown in Figure 5. The analysis in this paper will be based entirely on the images convolved to the resolution of the $5 \mathrm{GHz}$ images. At higher resolutions, imaging artifacts, that are not readily visible in the total intensity images, become apparent in spectral index images and in the absorption analysis, which are based essentially on differences. These artifacts are the result of the inadequate $(\mathrm{u}, \mathrm{v})$ coverage of the VLBA at high frequencies for a source of this complexity and size. Future observations will include additional antennas to address this difficulty. The $22 \mathrm{GHz}$ image was 
not made with the instrument's full resolution, knowing that it was to be convolved to the lower resolution of the lower frequency observations. Note that the $5 \mathrm{GHz}$ image, with contour levels that are deeper than those of Figure 2, shows the beginning of the extension, beyond 15 mas south of the core, that is seen at $2.3 \mathrm{GHz}$.

\section{Analysis}

The jet speed and angle results of WRB are based on the measured length ratio of the northern and southern features, on the long term expansion rate of the southern feature, and on the assumption that the northern and southern features both originated in the outburst of about 1959, so they have the same age. The 1995 data do not change the numbers. But the flux density ratio of the northern and southern features at $22 \mathrm{GHz}$ was 9 in the data from VRB, used by WRB. In the 1995 October $22 \mathrm{GHz}$ image, the ratio is about 3.7. The difference might be the result of the better coverage of short interferometer baselines in the 1995 data, or it might be the result of real changes in either the emission in the source or in the absorbing screen suggested by VRB and discussed below. By the beamed jet model of Blandford and Königl (1979), the flux density ratio $R$ and length ratio $D$ are related by $R=D^{\eta}$. The index $\eta$ is either $(2-\alpha)$ or $(3-\alpha)$, where $\alpha$ is the spectral index $\left(S \propto \nu^{\alpha}\right)$, for a continuous jet or a single component respectively. The data of VRB give $\eta=3.7$, which is consistent with the single component model and the spectral index observed in the southern feature. The 1995 data give $\eta=2.2$, which does not fit the simple model so well. Even with the continuous jet model, the spectral index would have to be flatter than is observed in the southern feature. But the flux density of the source has been decreasing with time for many years. In any one image, the northern feature is observed at an earlier time in its history than the southern feature because of light travel time effects - about 22 years earlier for the geometry of WRB and our assumed Hubble constant. In the early to mid 1970's, the integrated flux density of 3C 84 at $22 \mathrm{GHz}$ was roughly twice the the 1995 value (Nesterov, Lyuty, \& Valtaoja 1995). Therefore, while the distribution of the emission at $22 \mathrm{GHz}$ in the 1970's is not known, it is reasonable that the northern feature would be stronger ( $\eta$ lower) than would be expected by the simple, no-evolution beaming model.

The southern feature has a fairly uniform spectral index of $\alpha \approx-0.7$, a value rather typical of radio jets and hot spots in lobes. The northern feature is entirely different. It is far stronger at high frequencies and has spectral indices that vary both spatially and with frequency and that get as high as $\alpha>+4$. Even with less data, VRB argued that the steeply inverted spectral index, along with the extended size of the feature, effectively rules out other explanations than free-free absorption. Below we characterize and discuss this absorption. The core region also has an inverted spectrum and may be subject to some free-free absorption. However that spectrum could also simply be due to synchrotron self-absorption in the most compact regions of the jets. Therefore we do not include the core region in our discussion of free-free absorption.

Most of the analysis for this study was done with a special purpose program that operates 
on the CLEAN components from the AIPS images. Very deep CLEANs were done, so essentially all of the flux density is represented in the CLEAN components. The program is used to shift the components to align the images, make any required final amplitude calibration adjustments, convolve the components with a Gaussian beam roughly equivalent to the resolution of the lowest frequency image, and fit for the absorption. The fits are done both in the image plane and for points along a segmented slice as shown in Figures 1 and 2.

In order to image the absorption, it is necessary to align the images at different frequencies very accurately. Because of the presence of steep emission gradients, relative shifts of as small as 20 microarcseconds ( $\mu$ as) make small, but perceptible, changes in the distribution of the absorption. An alignment to that level of accuracy is desirable, but VLBI observations that don't utilize phase referencing retain no absolute position information. These observations were scheduled with occasional short scans on the nearby calibrator $0309+411$. Positions accurate to about a 1 mas could probably be obtained with the aid of these scans. But to obtain positions to $20 \mu$ as would require dual frequency observations to remove the effects of the ionosphere and scans all over the sky to calibrate the troposphere.

It is possible to use the source itself, in the case of 3C 84, to align the images very accurately. This requires the assumption that the southern feature is similar in structure at all frequencies - that there aren't large variations in observed spectral index. This assumption is supported by the images. The images could be aligned by finding the relative positions that minimize spectral index gradients. But a method that is at least as effective, and easier to automate, is to utilize the image cross correlation techniques previously developed for use on VLA data on 3C120 and M87 (Walker 1997 and references therein). The idea is to find the peak, as a function of position offset, of the cross correlation of portions of two images. For comparison, the software also does a least squares fit for the position offset of the image portions, generally obtaining similar results. For $3 \mathrm{C}$ 84, the regions greater than 5 mas south of the core were cross-correlated for all 6 possible pairs of images at each epoch. The images were shifted so as to minimize the offsets determined by the cross correlations. The 6 pairs over determine the problem, so the scatter of the results gives a crude estimate of the accuracy of the method. For each epoch, an alignment was found for which all reported offsets in RA and Dec were less than $5 \mu$ as, easily meeting our requirements. With these alignments, the uniformity of spectral indices was better than had been achieved previously with a tedious, non-automated procedure based directly on the spectral indices.

With alignment nominally accurate to a few microarcseconds, the internal motions of the source start to be a concern. Historically, the southern lobe is moving way from the core at about 0.3 mas $\mathrm{yr}^{-1}$. The counterjet would be expected to be moving at some fraction of that speed, probably lower by the length ratio of about 1.8. Thus we expect relative motions of jet and counterjet at the level of about $1.3 \mu$ as per day. The January observing sequence spanned 15 days and the October sequence spanned 13 days, so relative motions of up to $19 \mu$ as are expected. This has very little effect on the final results. Nevertheless an attempt has been made to account for it by moving the features more than 2 mas north of the core by an appropriate amount, depending 
on observing date relative to the day of the 5 and $8.4 \mathrm{GHz}$ observing. Rather than having an abrupt transition between the shifted and unshifted components, the region between -3.6 mas and +2 mas north of the core was stretched linearly. These distances are in proportion to the jet/counterjet length ratio.

The analysis program is capable of doing simultaneous fits for $22 \mathrm{GHz}$ amplitude $\left(I_{o}\right)$, emitted spectral index $\left(\alpha_{e}\right)$, absorption, and a covering factor $(f)$, all as a function of position. The equation of the fit is:

$$
I_{\nu}=I_{o} \times\left(\frac{\nu}{2.2 \times 10^{10}}\right)^{\alpha_{e}}\left[(1-f)+f e^{-\kappa}\right]
$$

where $I_{\nu}$ is the flux density measured at frequency $\nu(\mathrm{Hz})$, and $\kappa$ is the absorption coefficient, which is the main item of interest. The absorption is given by:

$$
\kappa=9.8 \times 10^{-3} L_{p c} n_{e}^{2} T^{-1.5} \nu^{-2}\left[17.7+\ln \left(T^{1.5} \nu^{-1}\right)\right]
$$

where $L_{p c}$ is the path length through the absorbing medium in parsecs, $n_{e}$ is the electron density in $\mathrm{cm}^{-3}, T$ is the temperature in degrees $\mathrm{K}$, and the terms in the square brackets are the Gaunt factor. The fits for absorption were actually done for the combination of parameters $\left(L_{p c} n_{e}^{2} T_{4}^{-1.5} g_{4}\right)$, where $T_{4}$ is the temperature in units of $10^{4} \mathrm{~K}$ and $g_{4}$ is the ratio of the Gaunt factor to the Gaunt factor for $T=10^{4} \mathrm{~K}$. Note that $L_{p c} n_{e}^{2}$ is simply the emission measure (EM), so for the case of a $10^{4} \mathrm{~K}$ constant temperature medium, the fit is simply for the emission measure.

The above parameterization has 4 unknowns. The observations reported here have 4 frequencies, and so have a maximum of 4 measurements at any point in the image or along the slice - sometimes fewer. Fitting for all 4 parameters was not really justified. After some experimentation, it was decided to fit only for $I_{o}$ and $\alpha_{e}$ along the southern feature, setting $E M=0$. On the northern feature, the subtle variations in emitted spectral index are overwhelmed by the strong absorption, so the fits were done for $I_{o}$ and absorption with $\alpha_{e}=-0.7$ and $f=1$. The results for the fits along the slice are shown in Figures 6 and 0 . The results for the absorption as a function of position in the image are shown as the gray scales in Figures 8 and 9 .

The quality of the fits is best assessed in Figures 6 and 7 . In those figures, the top panel shows the results for $\alpha_{e}$ and absorption along the slice, in terms of radial distance from the core. The amplitudes from the $22 \mathrm{GHz}$ slice are also shown for orientation. The lower panel shows the amplitudes from all 4 frequencies as lines and the fitted model amplitudes as crosses. Note the logarithmic scale and the very deep absorption, especially at $5 \mathrm{GHz}$. The fits are not perfect, in the sense that the absorption is not actually quite as sharp as for free-free absorption in a uniform medium. Better, even near perfect, fits can be obtained by releasing either $\alpha_{e}$ or $f$. Fitting for $\alpha_{e}$ gives spectral indices that are close to zero, which is significantly different from what is seen in the southern jet. We do not consider this to be very likely. Fitting for $f$ gives values in the range 0.98-0.995 - allowing less than $2 \%$ of the light to pass unabsorbed would give a near perfect fit. Probably more likely is that there are variations in the absorption over each beam area that account for the slightly flatter than a pure free-free spectrum. A detailed model has not been attempted and almost surely would not be unique. 


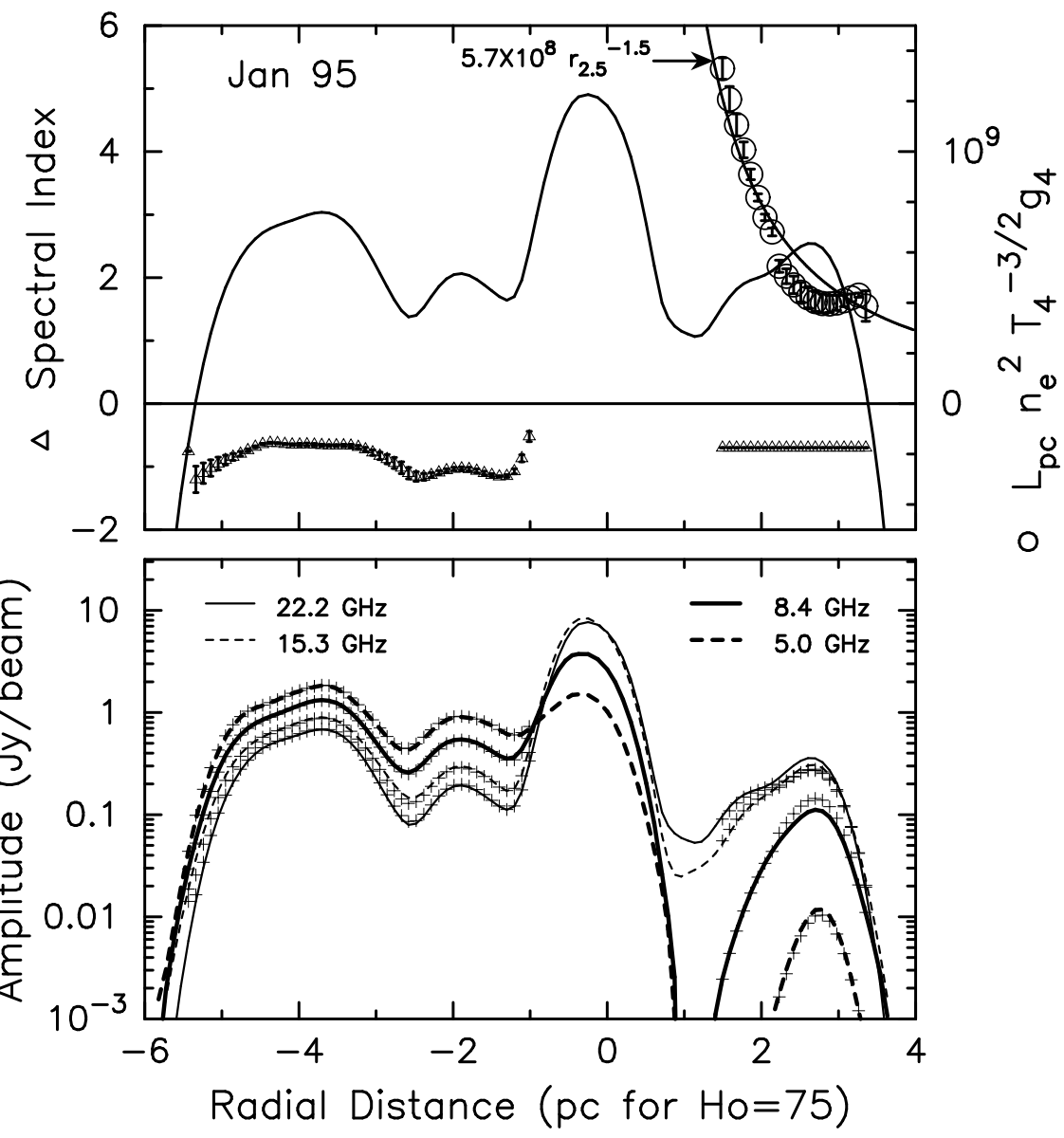

Fig. 6. - The results of least squares fits for emitted spectral index and absorption along the slice shown in Figure 1. The horizontal axis is the radial distance from the core (ie, not exactly along the slice) in parsecs assuming that $h=0.75$, for which $1 \mathrm{pc}$ is about 3 mas. The line in the top panel is the profile of flux density per beam at $22 \mathrm{GHz}$ while the lines in the bottom panel are the flux densities at 5.0, 8.4, 15.4, and $22 \mathrm{GHz}$, as labeled. Note the logarithmic flux density scale. The triangles in the top plot show the emitted spectral index, which is the result of a least squares fit for negative core distances (south) and is held fixed at $\alpha_{e}=-0.7$ to the north. The circles are the fitted absorption, parameterized as described in the text. Note that this parameterization becomes the emission measure if $T=10^{4} \mathrm{~K}$. The absorption is only fit to the north of the core - to the south there is no evidence for deviations from a simple spectral index. The formal error bars of the fits are shown. The curved line through the absorption results, and the equation at the top center of the plot, are the result of a fit of a power law to the absorption data. In the lower panel, the crosses are the data as predicted from the fit result at each point in the slice. Note that the step at about $1.8 \mathrm{pc}$ is at the edge of the region in which it was possible to use the $5.0 \mathrm{GHz}$ data in the fits. 


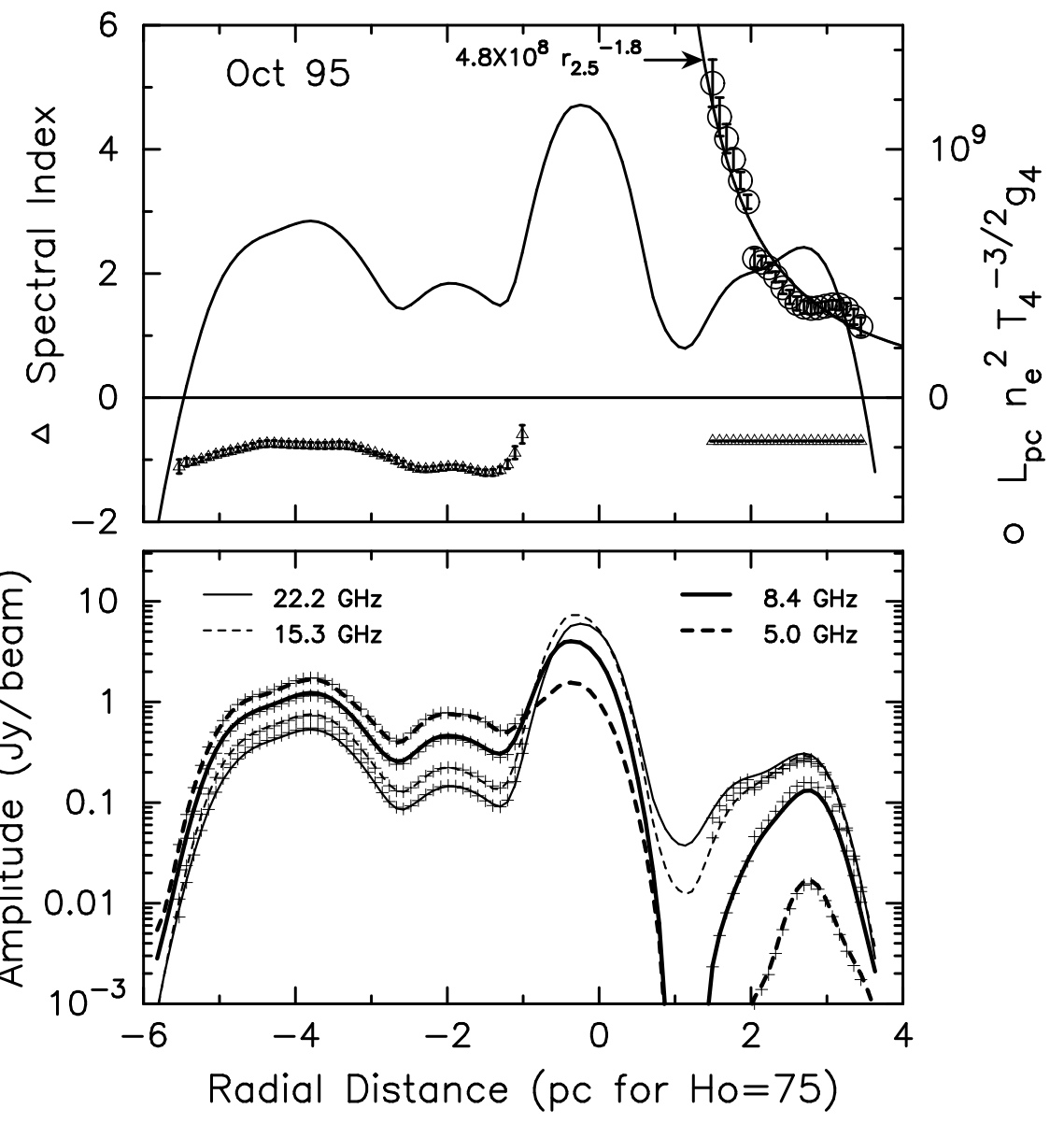

Fig. 7.- This is the same type of plot as Figure 6, but for the slice shown in Figure 2. Note the slightly lower absorption and corresponding somewhat higher flux density on the counter feature at low frequencies.

We are assuming that the origin of the jets, presumably in the vicinity of a massive black hole, is located at the northern end of the bright, compact regions seen at high frequency (Dhawan, Kellermann, and Romney 1998). We believe that this is consistent with the structures seen in our images, including the absence of any compact features farther north in the high resolution versions of the $43 \mathrm{GHz}$ images. But we cannot completely exclude the possibility that the true base of the jets is significantly north of this point as suggested by Nesterov, Lyuty, \& Valtaoja (1995) based on time lags between optical and radio variations. If the core is displaced northwards, the lack of strong radio emission at its location could be because the radio emission has not turned on in the inner jet, as suggested by Nesterov, Lyuty, \& Valtoaja, or because those inner regions are absorbed. The latter could be consistent with the fact that we do see structures in that region at the high frequencies, but not at lower frequencies, and with the possibility that the inverted spectrum in the region we call the core is due to absorption. A displaced core does not strongly 


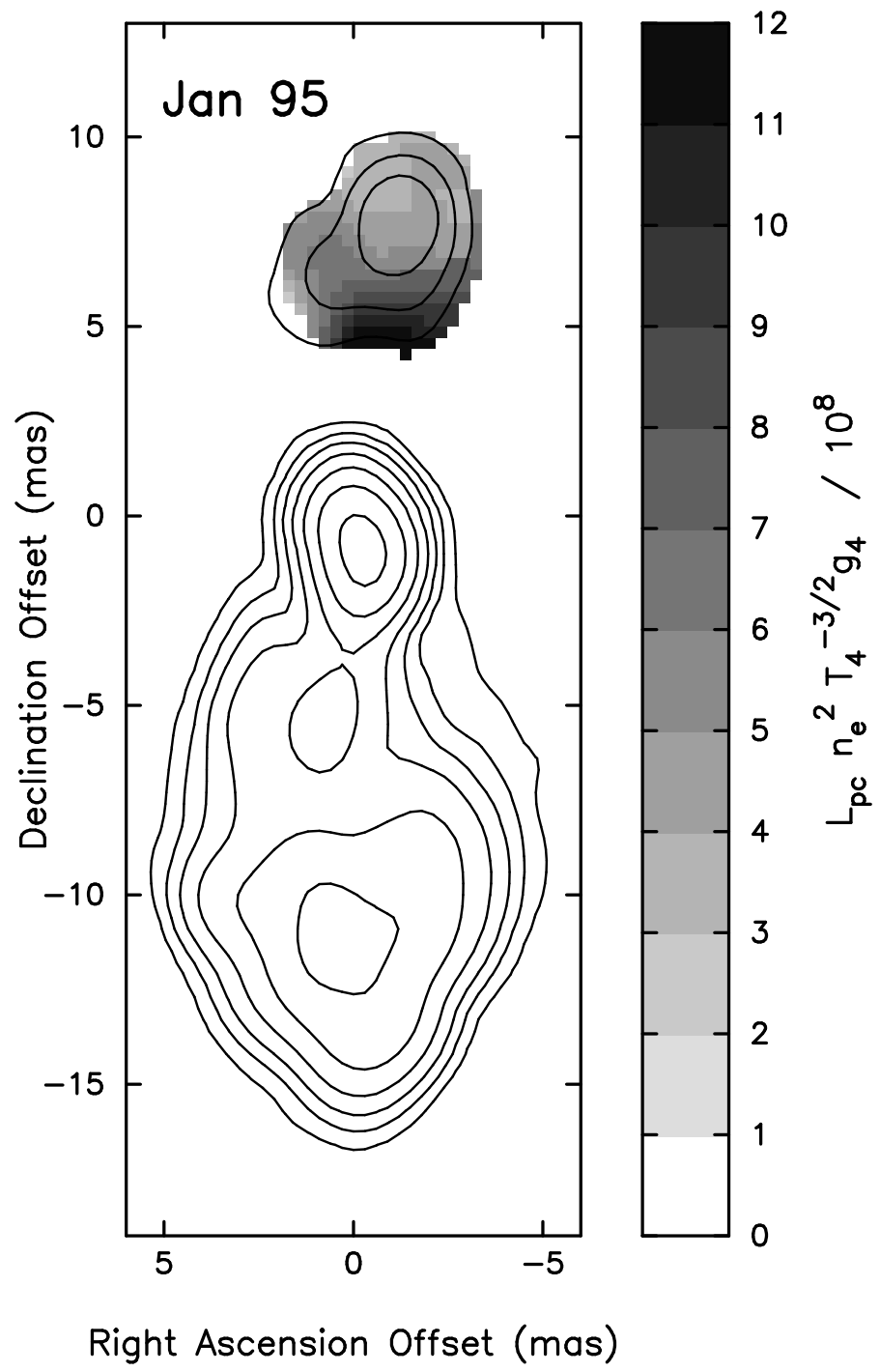

Fig. 8. - This image shows the two dimensional distribution of the free-free absorption in 3C 84 over the region of the counter feature in the 1995 January images. There is no detected absorption over the brighter southern feature. The absorption is parameterized in the same way as in Figure 6 . The overlayed contours are every third one from the 8.4 GHz image of Figure 1. The strong radial gradient away from the core is apparent.

affect the conclusions of this paper, but would affect the parameterization of the radial gradient of absorption. It would also affect the geometry derivation of WRB in the sense that the jets would lie closer to the line-of-sight and the simple relativistic beaming model would not work quite as well.

Our fit results show that the absorption has a strong gradient away from the core. This is seen clearly in the slices of Figures 6 and 7 and in the gray scale images of Figures 8 and 9 . An 


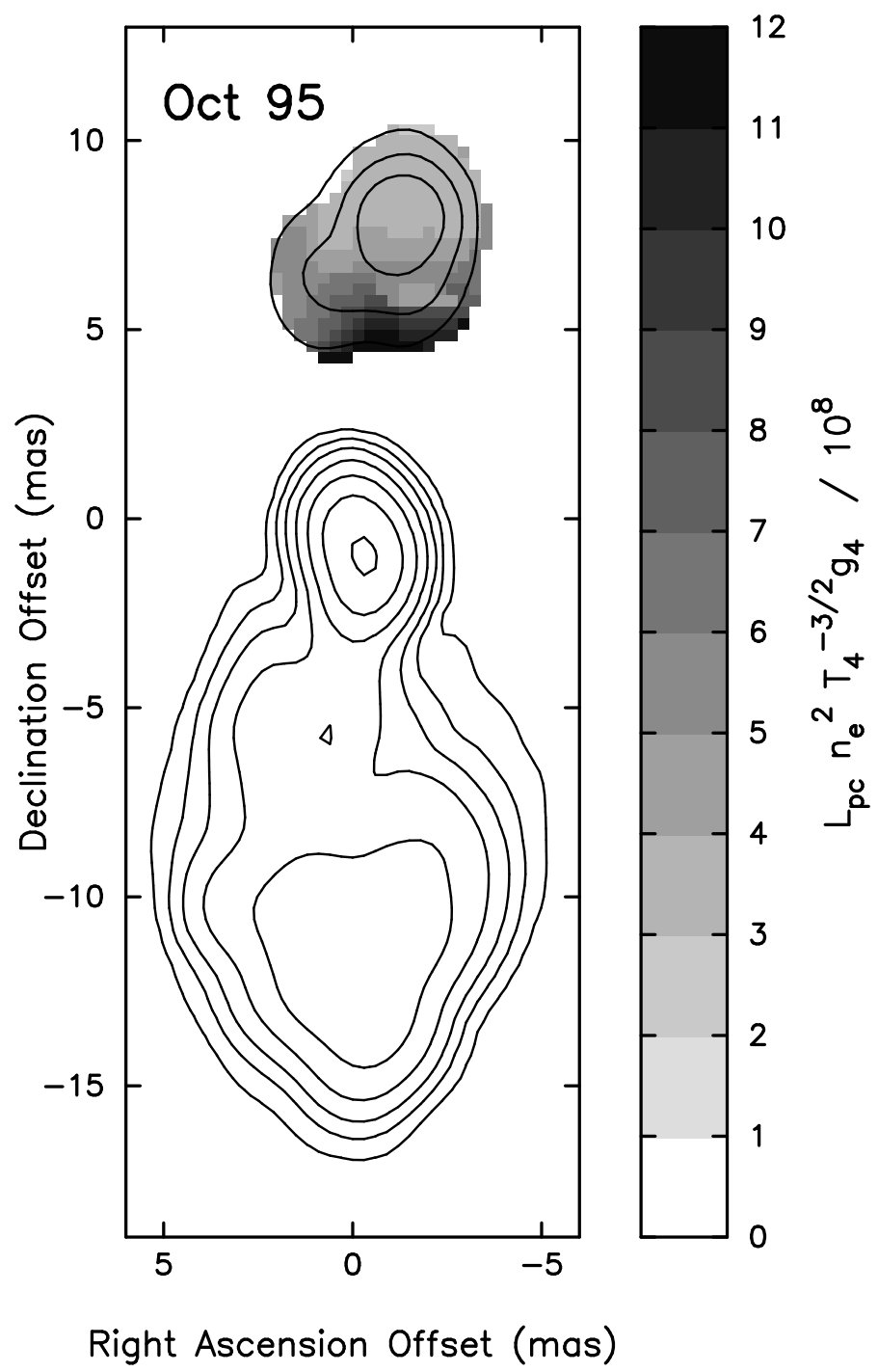

Fig. 9. - This is the same information as Figure 8, except that it is for the 1995 October data. The time interval between the two data sets is really too short to trust measured changes, but there are indications that the absorption has decreased between January and October.

attempt to characterize this gradient was made using the slice results by fitting a power law in $r_{2.5}$, the core distance in units of $2.5 \mathrm{pc}$. This reference distance was chosen so that the constant in the equation corresponds to the value at about the middle of the northern feature. The results are displayed on Figures 6 and 0 . They are:

$$
L_{p c} n_{e}^{2} T_{4}^{-1.5} g_{4}=5.7 \times 10^{8} r_{2.5}^{-1.5}
$$

in January and

$$
L_{p c} n_{e}^{2} T_{4}^{-1.5} g_{4}=4.8 \times 10^{8} r_{2.5}^{-1.8}
$$


in October. The power law is clearly only a rough description of the results. The formal fit errors for the exponent are less than 0.1. But it is clear that there are large deviations from any power law, so the value of the exponent should not be taken too seriously. A wider range of core distances would be useful for any characterization of the gradient.

The feature $(\mathrm{NN})$, seen at about 80 mas north of the core at low frequencies by STV, also has a peaked spectrum that STV conclude is the result of free-free absorption. For this feature, the absorption is much weaker since it occurs at a much lower frequency. The simple power laws fit to our higher frequency data in the region less than 10 mas from the core, if extrapolated to the more distant feature, would give far too much absorption. If the power law characterization is reasonable, the exponent must be in the vicinity of the -2.6 determined by STV. But a power law with a -2.6 exponent is not very consistent with our data in the 4 to 10 mas range. It is likely that the amount of ionized gas eventually falls off faster between 10 and 80 mas (3.2 and 26 pc) than it does over the 4 to 10 mas (1.3 to $3.2 \mathrm{pc}$ ) range over which we measure it. In fact, the existence of structure even closer to the core at 15 and $22 \mathrm{GHz}$ (see Figure ) suggests that the absorption does not continue to increase as fast with decreasing core distance inside the region of our measurements as it does further out.

\section{Discussion}

The current data provide more frequencies than were available for previous discussions of the spectrum of the northern feature and provide measurements of all frequencies taken at approximately the same time, so that time variability should not be a source of uncertainty. Based on the earlier 8.4 and $22 \mathrm{GHz}$ data, with a 2 year separation, VRB and LLV argue that the inverted spectrum of the northern feature is not synchrotron self-absorption on the grounds that, given the spectrum and the size of the feature, the magnetic field energy density would have to be greater than the particle energy density by a factor of $10^{14}$, which is unlikely. Also the total energy content of the jet with the implied magnetic field would be unreasonably high. The current measurements provide a rather simpler argument. The observed spectral index on the northern feature between 5.0 and $8.4 \mathrm{GHz}$ is about $\alpha=4$. This is significantly steeper than the optically thick synchrotron spectral index of 2.5. Therefore, synchrotron self-absorption cannot be the only cause of the inverted spectrum. LLV also consider stimulated Raman scattering as the cause of the inverted spectrum, but rule it out on the grounds that the brightness temperature is inadequate. That argument remains unchanged. The Razin-Tsytovich effect, which exponentially suppresses synchrotron radiation at frequencies below those where the phase velocity is greater than c, can cause a steeply inverted spectrum below $\nu_{r} \simeq 20 n_{e} / B \mathrm{~Hz}$. But with only $\nu^{-1}$ in the exponent, compared to the $\nu^{-2}$ of free-free absorption, it cannot produce the change from about $\alpha=0$, seen between 15 and $22 \mathrm{GHz}$, to about $\alpha=4$, seen between 5.0 and $8.4 \mathrm{GHz}$, without an unphysically steep underlying emitted spectral index. Also, the Razin-Tsytovich effect operates in the emitting region rather than along the line-of-sight, so it is difficult to understand why there would be a 
very big difference between the northern and southern features. Free-free absorption, on the other hand, fits the spectra reasonably well, and, to the extent that there are deviations, they are what might be expected from non-uniformities in the medium, something that is reasonable to expect.

The opacity fits presented above give an emission measure of about $5 \times 10^{8}$ at $2.5 \mathrm{pc}$ with a fairly steep radial gradient, assuming a temperature of $T=10^{4} \mathrm{~K}$. The path through the absorbing medium cannot be significantly larger than the projected distance of the line-of-sight from the core. In fact, if the geometry were that of a thin disk, the path would be significantly smaller than the projected offset. The density of ionized gas required is roughly $n_{e}=2 \times 10^{4} \mathrm{~cm}^{-3}$ for a thick $(1 \mathrm{pc})$ medium at $10^{4} \mathrm{~K}$, rising to significantly higher values for a thinner medium or a higher temperature.

LLV point out that the Thomson optical depth along the line-of-sight must not significantly exceed unity, constraining the density to be

$$
n_{e} \lesssim 5 \times 10^{5} L_{p c}^{-1} \mathrm{~cm}^{-3}
$$

This density is not all that far from that implied by the free-free absorption measurements. If we insert the inequality of Equation 5 into Equations 3 and 4, we get:

$$
T \lesssim 5.8 \times 10^{5} r_{2.5} L_{p c}^{-2 / 3}
$$

for January and

$$
T \lesssim 6.5 \times 10^{5} r_{2.5}^{1.1} L_{p c}^{-2 / 3}
$$

for October. The fact that $L_{p c}$ is not well constrained leaves room for a fairly wide range of temperature limits. But for values of $L_{p c}$ between 0.01 and 1 , the temperature at $2.5 \mathrm{pc}$ cannot exceed between about $1.4 \times 10^{7}$ and $6.5 \times 10^{5}$ respectively in October and slightly less in January. These limits don't really cause any problems for models that we are aware of, but it is clear that the temperature cannot greatly exceed the $10^{6} \mathrm{~K}$ called for in some cases (eg. Königl and Kartje 1994; Gallimore, Baum, \& O’Dea 1997)

The region where free-free absorption is observed is a few parsecs from the central object in NGC 1275. LLV estimate the bolometric luminosity to be about $4 \times 10^{44} \mathrm{ergs} \mathrm{s}^{-1}$, which is about the Eddington luminosity for a $10^{6} M_{\odot}$ black hole. If that is the mass of the central object, a location $2.5 \mathrm{pc}$ away is at roughly $3 \times 10^{7} R_{G}$ (The gravitational radius $R_{G}=2 G M / c^{2}$ ). On the other hand, the same location would only be about $3 \times 10^{4} R_{G}$ from a $10^{9} M_{\odot}$ black hole. These distances are in the outer regions typically covered in accretion disk models, if not well beyond. In such regions, models are complicated by the possible importance of self gravity and of external illumination of unknown geometry. The temperature at these distances is expected to be too low for ionization without illumination by ionizing radiation from the central regions. Such illumination requires an appropriate geometry such as a flaring or warped disk or a source of radiation offset from the disk center, possibly from a jet or from scattering. LLV give an extensive discussion of the implications for disk models of the observation of free-free absorption in NGC 1275 - a discussion that is not significantly changed by the new data. 
The idea that AGN disks are likely to be illuminated by ionizing radiation from the central compact region near the black hole has been explored as part of efforts to model the spectra of

broad line regions and the structure of outer disks (see Collin and Huré 1999 and references therein including Collin-Souffrin \& Dumont 1990). Generally the density of the outer disk is expected to be much higher than implied by the free-free absorption. However, much of the disk is sufficiently optically thick that the central regions are shielded from ionizing radiation. These regions will be neutral and will not contribute to the free-free absorption or to many of the observed optical emission lines. But there is likely to be an ionized "chromosphere" above the dense regions. At large radii, the density is too low to provide shielding and the disk is fully ionized and at a temperature of about $7000 \mathrm{~K}$. This chromosphere, or the ionized regions at large distances, could be the absorbing region.

An alternative to the irradiated disk for the location of the ionized material responsible for the free-free absorption might be a wind off the surface of the disk. For example, Königl and Kartje (1994 — see also other references therein) discuss the properties of centrifugally driven winds in the AGN context. Winds of this type are the leading model for the origin of the bipolar outflows that are very common in young stellar objects. They seem likely to exist whenever a disk is threaded by magnetic fields - a situation that is probably hard to avoid. The temperatures and densities of some of the examples presented in Königl and Kartje are of the magnitude required by our data.

It seems likely that both of the above scenarios apply in an AGN. The central regions are a strong source of ionizing radiation which is very likely to affect some portion of the disk. And, given the almost certain presence of magnetic fields in any accretion disk, combined with the ubiquitous presence of magnetically driven winds from galactic examples of accretion disks, it seems hard to avoid having a magnetically driven wind. There is a considerable range of possible variations on these models, depending on the black hole mass, the accretion rate, the disk composition, the magnetic field strength, and other factors, so a variety of types of data will be required to determine anything like a unique model. Because of the large range of possible models, we have not attempted detailed comparisons with any specific models. But our data do provide some firm constraints that any model must match. We are attempting to obtain additional constraints by making multi-frequency observations that can be used at higher resolution than those presented here. Such observations will also be sensitive to temporal variations in the absorption since 1995 . In addition, we are searching for recombination lines from the absorbing medium.

\section{Conclusions}

The primary conclusions of this work are:

- The suggestion from VRB and WRB, based on two frequencies and observations separated by 2 years, that there is free-free absorption of the northern feature in 3C 84 at a few 
parsecs from the central object, is confirmed. We present two separate epochs in which the absorption was observed nearly simultaneously at 5 frequencies. The observed spectral indices are sufficiently steep to preclude other absorption mechanisms.

- The free-free absorption shows a two dimensional structure dominated by a gradient with distance from the radio core. The absorption is greater near the core and falls off with distance. If the absorption goes as a power law with distance, the exponent is a bit above -2 over the range of the observations presented here (about 1.5 to 3.5 pc projected distance from the core). However a somewhat steeper exponent is required to match up with the observations of STV at around $25 \mathrm{pc}$. Those observations were taken at the same time as ours.

- The observed absorption is consistent with the model proposed by WRB and VRB. In that model, the northern feature is on the far side of the system relative to the Earth. There is an accretion disk extending to the parsec scales observed here and that accretion disk has associated ionized gas that is responsible for the absorption. The amount of ionized gas falls off fairly rapidly with core distance.

Various models, including disk ionization by radiation from the central regions and disk-driven hydromagnetic winds, might provide the necessary ionized material in a geometry that would only affect the far-side jet. The free-free absorption results provide firm constraints on the ionized gas on parsec scales, including positional information, that any model of the central regions of NGC 1275 must match.

We would like to thank J. Benson and W. Alef for their contributions to these observations. We also thank J. Wrobel and G. Taylor for useful discussions. Finally, we thank the staff of the VLBA for their invaluable support. These observations would not have been possible without the major advances in frequency flexibility and image quality provided by the VLBA. The National Radio Astronomy Observatory is a facility of the National Science Foundation, operated under cooperative agreement by Associated Universities, Inc.

\section{REFERENCES}

Allen, S. W., \& Fabian, A. C. 1997, MNRAS, 286, 583

Biretta, J. A., Bartel, N., \& Deng, J. 1991, in IAU Coll. 131, Radio Interferometery: Theory, Techniques, and Applications, eds. T. J. Cornwell \& R. A. Perley (San Francisco: Astronomical Society of the Pacific), ASP Conference Series, Vol. 19, 334

Blandford, R. D. \& Königl, A. 1979, ApJ, 334, 560

Brodie, J. P., Schroder, L. L., Huchra, J. P., Phillips, A. C., Kissler-Patig, M., \& Forbes, D. A. 1998, AJ, 116, 691 
Carlson, M. N. et al. 1998, AJ, 115, 1778

Collin-Souffrin, S. \& Dumont, A. M. 1990, A\&A, 229, 292.

Collin, S. \& Huré, J.M. 1999, A\&A, 341, 385.

Dent, W. A. 1966, ApJ, 144, 843

DeYoung, D. S., Roberts, M. S., and Saslaw, W, C. 1973, ApJ, 185, 809.

Dhawan, V., Kellermann, K. I., \& Romney, J. D. 1998, ApJ, 498, L111

Gallimore, J. F., Baum, S. A., and O'Dea, C. P. 1997, Nature, 388, 852

Jones, D. L. et al. 1996, ApJ, 466, L63

Jones, D. L. \& Wehrle, A. E. 1997, ApJ, 484, 186

Kaisler, D., Harris, W. E., Crabtree, D. R., \& Richer, H. B. 1996, AJ, 111, 2224

Kellermann, K. I., Vermeulen, R. C., Cohen, M. H., and Zensus, J. A. 1999, BAAS, 31, 856

Königl, A. \& Kartje, J. F. 1994, ApJ, 434, 446

Krichbaum, T. P., Witzel, A., Graham, D. A., Schalinski, C. J., \& Zensus, J. A. 1993, in Sub-arcsecond Radio Astronomy, eds. R. J. Davis \& R. S. Booth (Cambridge: Cambridge University Press), p. 181

Lazareff, B., Castets, A, Kim, D.-W., \& Jura, M. 1989, ApJ, 336, L13

Levinson, A., Laor, A., \& Vermeulen, R. C. 1995, ApJ, 448, 589

Marr, J. M., Backer, D. C., Wright, M. C. H., Readhead, A. C. S., \& Moore, R 1989 ApJ, 337, 671

Minkowski, R. 1957, in IAU Symposium No. 4, Radio Astronomy, ed. H. C. van de Hulst (Cambridge: Cambridge University Press), p. 107

Napier, P. J., Bagri, D. S., Clark, B. G., Rogers, A. E. E., Romney, J. D., Thompson, A. R., \& Walker, R. C. 1994, Proc. IEEE, 82, 658

Nesterov, N. S., Lyuty, V. M., \& Valtaoja, E. 1995, A\&A, 296, 628

Nørgaard-Nielsen, H. U., Goudfrooij, P., Jørgensen, H. E., \& Hansen, L. 1993, A\&A, 279, 61

O’Dea, C. P., Dent, W. A., Balonek, T. J. 1984, ApJ, 278, 89

Pauliny-Toth, I. I. K. \& Kellermann, K. I. 1966, ApJ, 146, 634

Pauliny-Toth, I. I. K., et al. 1976, Nature, 259, 17

Pedlar, A., Ghataure, H. S., Davies, R. D., Harrison, B. A., Perley, R., Crane, P. C., \& Unger, S. W. 1990, MNRAS, 246, 477

Readhead, A. C. S., Hough, D. H., Ewing, M. S., Walker, R. C., \& Romney, J. D. 1983, ApJ, 265, 107

Romney, J. D. 1978, Ph. D. thesis at the California Institute of Technology 
Romney, J. D., Alef, W., Pauliny-Toth, I. I. K., Preuss, E., \& Kellermann, K. I. 1982, in IAU Symposium no. 97: Extragalactic Radio Sources, eds. D. S. Heeschen \& C. M. Wade (Dordrecht: Reidel) p. 291.

Romney, J. D., Alef, W., Pauliny-Toth, I. I. K., Preuss, E., \& Kellermann, K. I. 1984, in IAU Symposium No. 110: VLBI and Compact Radio Sources, eds. R. Fanti, K. Kellermann, \& G. Setti (Dordrecht: D. Reidel Publishing Company), p. 137

Romney, J. D., Kellermann, K. I., \& Alef, W. 1999 in preparation

Shakura, N.I. \& Sunyaev, R. A. 1973, A\&A, 24, 337

Shepherd, M. C., Pearson, T. J., \& Taylor, G. B. 1995, BAAS, 26, 987

Sijbring, L, G. 1993, thesis at the University of Groningen

Silver, C. S., Taylor, G. B., \& Vermeulen, R. C. 1998 (STV), ApJ, 502, 229

Taylor, G. B., \& Vermeulen, R. C. 1996, ApJ, 457, L69

Ulvestad, J. S., Wrobel, J. M., \& Carilli, C. L. 1999, ApJ, 516, 127.

Venturi, T., Readhead, A. C. S., Marr, J. M., and Backer, D. C. 1993, ApJ, 411, 552

Vermeulen, R. C., Readhead, A. C. S., \& Backer, D. C. 1994 (VRB), ApJ, 430, L41

Walker, R. C., Romney, J. D., \& Benson, J. M. 1994 (WRB), ApJ, 430, L45

Walker, R.C., Romney, J.K., Vermeulen, R.C., Dhawan, V., \& Kellermann, K.I. 1996, in Extragalactic Radio Sources; IAU Symposium 175, eds. R. Ekers, C. Fanti, and L. Padrielli (Dordrecht: Kluwer), 30

Walker, R. C. 1997. in Accretion Phenomena and Related Outflows; IAU Colloquium 163, eds. D. T. Wickramasinghe, G. V. Bicknell, and L. Ferrario (San Francisco: Astronomical Society of the Pacific), 591

Walker, R.C. 1997, ApJ, 488, 675

Walker, R. C., Kellermann, K. I., Dhawan, V., Romney, J. D., Benson, J. M., Vermeulen, R. C., \& Alef, W. 1998. in Radio Emission from Galactic and Extragalactic Compact Sources; IAU Colloquium 164, eds. J. A. Zensus, G. B. Taylor, and J. M. Wrobel (San Francisco: Astronomical Society of the Pacific), 133 